\title{
Borderline malignant change in recurrent müllerian papilloma of the vagina
}

\author{
S P Dobbs, P A V Shaw, L J R Brown, D Ireland
}

\begin{abstract}
Malignant change occurred in a benign, recurrent vaginal müllerian polyp. The patient, a 49 year old woman with cerebral palsy, presented with a polypoid mass in the vagina. At four years of age she had presented with a haemorrhagic polyp, and over the following years she had recurrent irregular bleeding and regrowth of the polypoidal mass, requiring a total of 10 operations to excise the polyp. Histological examination of the specimen showed typical müllerian features with tubal, endometrioid, and endocervical cell types. There were significantly abnormal nuclei, indicating low grade or borderline malignancy. Review of previous biopsies showed similar müllerian features but no atypia. This is the first reported case of borderline malignant change in a previously benign recurrent müllerian papilloma of the vagina. Definitive radical surgery or radiotherapy is contraindicated in this patient and she remains under follow up. (F Clin Pathol 1998;51:875-877)
\end{abstract}

Keywords: müllerian papilloma; vagina; borderline malignant change

Tumours of the vagina are rare. Malignant lesions account for only $3-5 \%$ of all gynaecological cancers and of these over $80 \%$ are squamous cell carcinomas. ${ }^{1}$ Benign tumours are even less common and do not usually progress to malignancy.

The epithelium of the upper part of the vagina is thought to be of müllerian origin and has the potential for glandular as well as squamous differentiation. Occasionally glandular tumours may arise in this area. ${ }^{2}$ Classically these are malignant and may have been associated with fetal exposure to diethylstilboestrol. ${ }^{3}$ However, rare benign growths unrelated to diethylstilboestrol have also been reported. These have a branching, papillary architecture and are covered by a variable cuboidal or columnar epithelium. Various names have been attached to these lesions, but as electronmicroscopy and immunohistochemical investigations confirm typical müllerian features of glandular structure, microvilli, and cytokeratins, ${ }^{45}$ müllerian papilloma seems the most appropriate designation.

Mullerian papillomas characteristically arise in childhood and are cured by simple excision. There are isolated reports of cases in adults but only one reported case of tumour recurrence in an otherwise typically benign lesion. ${ }^{5}$ To our knowledge this is the first report of a recurrent benign müllerian papilloma which developed to show borderline malignant features.

\section{Case report}

A 49 year old nulliparous woman with cerebral palsy was referred to the gynaecological oncology department with a long history of irregular vaginal bleeding and a polypoid mass in the vagina.

She originally presented at the age of four years to the casualty department with heavy vaginal bleeding. On examination under anaesthetic a large bleeding polyp was noted on the anterior vaginal wall. This was ligated and removed. Throughout the following 30 years the patient was seen intermittently at the gynaecological department with recurrent irregular bleeding and regrowth of the polyp. She underwent 10 further operations to excise the polypoid mass.

After a gap of 10 years the patient was referred again with a history of intermittent bleeding and discharge. A further examination under anaesthetic revealed a large fleshy, clinically malignant mass in the upper anterior third of the vagina. There was no evidence of paravaginal or pelvic spread of the tumour, and the cervix appeared normal under colposcopic examination. Complete excision was not possible and further biopsies were taken.

\section{Pathological findings}

The first specimen from 1955 was described as "hidradenoma papilliferum" and was not available for review. Subsequent reports describe the tumour variously as a polypoidal mass with simple glandular structures, ectopic endocervical glands within the vagina, or dismissed as a slightly unusual but benign endocervical polyp. Review of the available material dating from 1974 onwards revealed at least some areas resembling the diagnostic biopsy but no evidence of architectural or cytological atypia to suggest malignant change.

The diagnostic biopsy consisted of papillary fragments $20 \mathrm{~mm}$ in maximum dimension. Microscopy showed delicate papillary fronds consisting of a loose oedematous stroma covered by a mixture of müllerian epithelia including recognisable tubal, endometrial, and endocervical types, as well as intermediate forms (fig 1). Rounded calcified bodies were seen within the connective tissue. Other parts had a more solid appearance reminiscent of a simple endocervical polyp in areas with variably inflamed squamous epithelium.

Some of the fronds were covered with a multilayered epithelium, while a few lacked connective tissue cores. On closer examination the
Accepted for publication 7 July 1998 


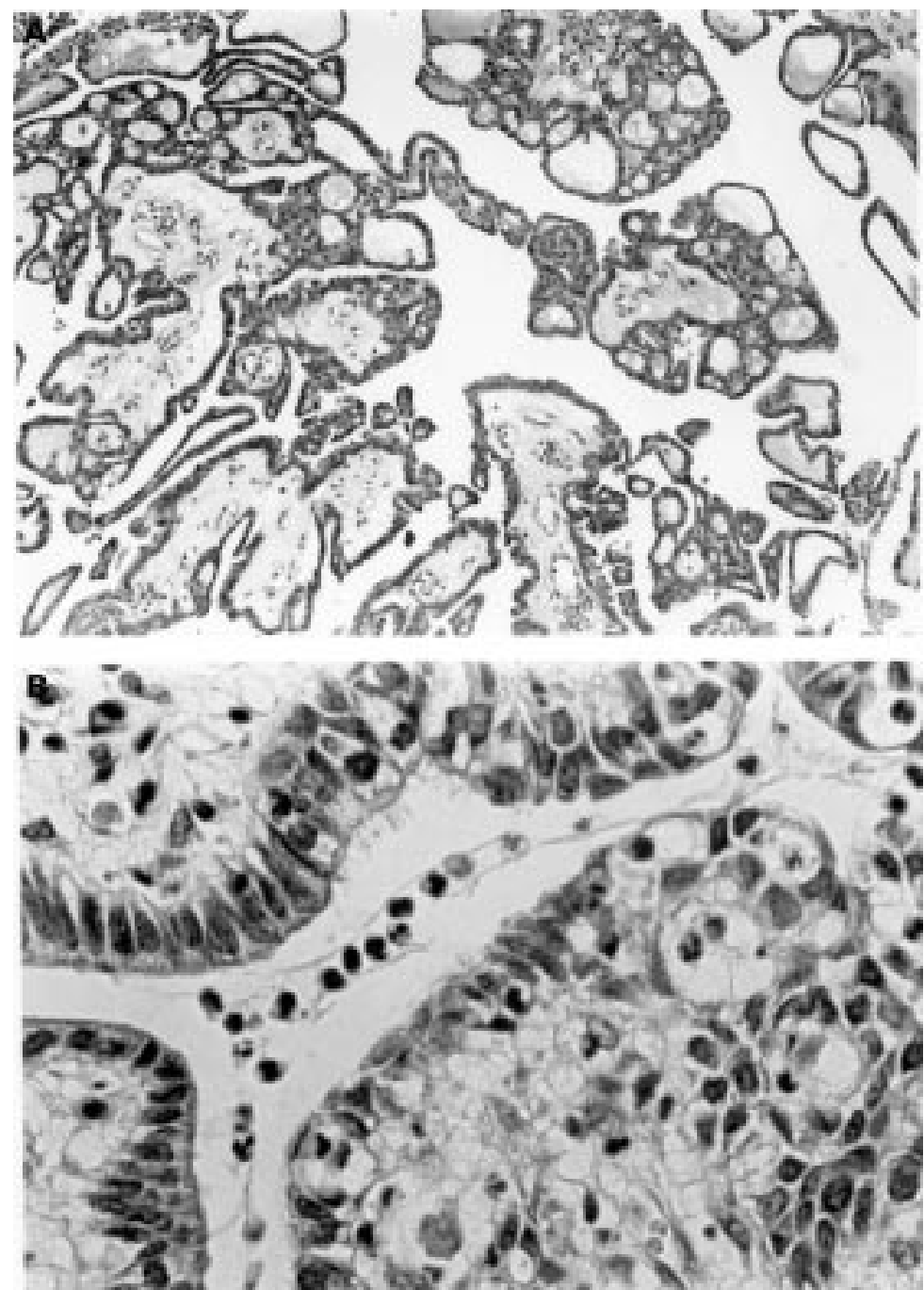

Figure 1 (A) Low power view of polyp showing architecture with predominantly regular epithelium. (B) High power view showing different epithelial types (tubal, endocervical, and simple).

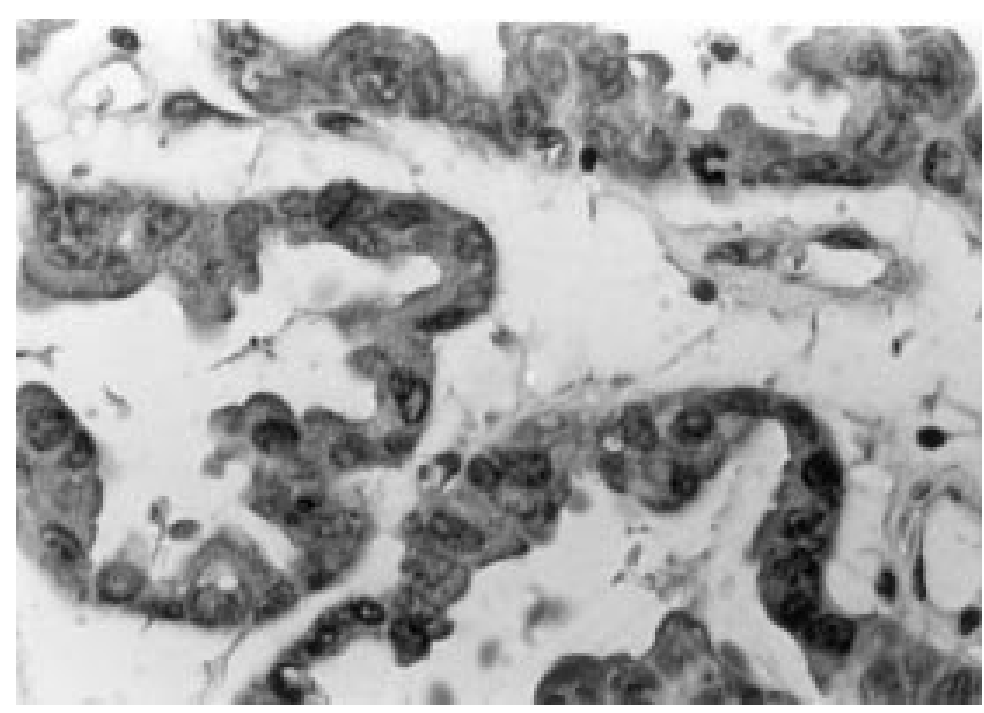

Figure 2 High power view of polyp showing atypical epithelium with stratification, nuclear variation, small papillae, and an abnormal mitotic figure. epithelial nuclei in these areas showed significant nuclear atypia with frequent mitoses, including atypical forms (fig 2) No invasion was identified, but the fragmentary nature of the specimen made adequate assessment impossible. Review of the previous biopsies failed to show any similar atypical areas.

By analogy with ovarian tumours, these abnormal epithelial changes were interpreted as indicating at least borderline malignancy. Future behaviour of the tumour was thought difficult to predict, apart from the obvious observation that it was likely to recur.

\section{Discussion}

We describe a unique case of malignant progression in a müllerian papilloma. The vagina is formed by fusion of the urogenital sinus with the müllerian ducts. It is thought that the upper three fifths of the vagina originate from the müllerian epithelium and the lower two fifths from the urogenital sinus. ${ }^{2}$ As the vagina is ultimately lined by squamous epithelium, the originally glandular müllerian epithelium (originating from endoderm) undergoes metaplasia during development. Tumours of the vagina can originate from either the urogenital sinus or the müllerian ducts. In 1971 Graf and Pfleiderer ${ }^{6}$ reported a vaginal adenoma and concluded that it had originated from müllerian epithelium. The light microscopy findings in other reported cases of papillary tumours of the upper vagina or cervix $^{7-9}$ have shown papillae covered with columnar or squamous epithelium and with a central fibrovascular core. There has been much debate on the origins of these papillary tumours, though the light microscopy features are essentially the same. Both endometrial type squamous metaplasia and extracellular and intracellular mucopolysaccharides have been described. ${ }^{45}$ Neither papillomata nor the characteristic hobnail epithelium found in mesonephric epithelium have been observed in mesonephric remnants. ${ }^{10}$ Immunohistochemical studies on a vaginal papilloma by Luttges and Lubke $^{5}$ showed positive reactions for type 1 cytokeratin, epithelial membrane antigen (EMA), and carcinoembryonic antigen (CEA). Similar findings are present in endometrial glands and epithelium. The tumour here did not express type II cytokeratin or vimentin, which are typically found in mesothelial cell types. Ulbright et al performed electronmicroscopy on a papillary vaginal tumour and showed ultrastructural features identical to müllerian epithelium; in particular they demonstrated microvilli on the luminal borders of glands, lysosomes, squamous metaplasia, and pseudoinclusions of collagen. ${ }^{4}$

The majority of vaginal papillomas occur early in childhood, typically presenting before 10 years of age with vaginal bleeding. There is one reported case of recurrence two years after primary treatment, ${ }^{5}$ possibly owing to incomplete excision of the primary tumour. In our case there were 10 documented recurrences over 40 years. Though the earliest pathology slides are not available, it is likely that all these recurrences arose from the tumour that was present when 
the patient presented initially at the age of four years. The most recent biopsy of the tumour showed atypical epithelial changes indicating low grade or borderline malignancy. On histological review of the previous biopsies there is no evidence of any malignant transformation.

The differential diagnosis in this woman would include vaginal adenocarcinoma. However, the morphology of the epithelium is not that of clear cell adenocarcinoma and there is no record of diethylstilboestrol exposure.

Perhaps more credible is spread of a cervical tumour to the vagina. The appearances were not those of the usual endocervical adenocarcinoma but are similar to villoglandular papillary carcinoma or the rare pure papillary carcinoma. Villoglandular papillary carcinoma is a low grade carcinoma, typically with a villous morphology overlying invasive glandular structures. However, the villi of villoglandular carcinoma are broader and unbranched and are covered by epithelium of recognisably endocervical type and not by the mixture of types seen in this case. Pure papillary carcinoma is usually high grade and is not found with a benign component. The history of similar benign papillary tumour over many years before the development of malignant change would be against spread from the cervix or elsewhere.
We believe this is the first reported case of borderline malignant transformation of a previously benign vaginal müllerian papilloma. In this patient the associated cerebral palsy was considered a contraindication to definitive surgery or radical radiotherapy. The patient remains under follow up.

1 Sulak P, Barnhill D, Heller P, et al. Non squamous cancer of the vagina. Gynecol Oncol 1988;29:309-20.

2 Forsburg JG. Cervicovaginal epithelium: its origin and development. Am f Obstet Gynecol 1973;113:1025-43.

3 Herbst AL. Clear cell adenocarcinoma and the current status of diethylstilboestrol exposed females. Cancer 1981;48: 484-9.

4 Ulbright TM, Alexander RW, Kraus FT. Intramural papilloma of the vagina: evidence of müllerian histogenesis. Cancer 1981;48:2260-5.

5 Luttges JE, Lubke M. Recurrent benign müllerian papilloma of the vagina. Immunohistochemical findings and histogenesis. Arch Gynecol Obstet 1994;255:157-60.

$6 \mathrm{Graf} \mathrm{H}$, Pfleiderer A. Gland forming benign vaginal tumour: histochemical structures on pathogenesis. Arch Gynakol 1971;211:475-83.

7 James T. A benign polypoid tumour of the cervix uteri in a girl three years old. F Obstet Gynaecol Br Emp 1951;58:7623 .

8 Selzer I, Nelson HM. Benign papilloma (polypoid tumour) of the cervix uteri in children. Am $\mathcal{F}$ Obstet Gynecol 1962;84:165-9.

9 Janovski NS, Kasdon EJ. Benign mesonephric papillary and polypoid tumors of the cervix in childhood. F Pediatr 1963; 63:211-16.

10 Norris HJ, Begley GP, Taylor HB. Carcinoma of the infant vagina. A distinctive tumour. Arch Pathol 1970;90:473-9. 